\title{
Zonotopic Extended Kalman Filter For RUL Forecasting With Unknown Degradation Behaviors
}

\author{
Ahmad Al-Mohamad ${ }^{\dagger, \ddagger}$, Vicenç Puig ${ }^{\dagger}$ and Ghaleb Hoblos ${ }^{\ddagger}$
}

\begin{abstract}
This paper proposes a novel approach for Remaining Useful Life (RUL) forecasting using interval modelbased prognostics techniques based on zonotopes without prior knowledge of the degradation behaviors of the system. Although Kalman filtering techniques have proved their estimation ability with Gaussian noises, an interval approach with zonotopic sets technique has been integrated for optimal estimation of parameters with unknown-but-bounded noises. Moreover, the proposed model-based prognostics technique has been applied to a DC-DC converter described as a nonlinear dynamical system affected by degradation behaviors. Thus, the estimated degraded parameters are adopted in the RUL prediction technique that propagates the zonotopic sets until the Endof-Life (EoL) of the system. In general, the technique is split into estimation and prediction phases using Zonotopic Extended Kalman Filter (ZEKF) to deal with the nonlinearities of the system and compute the optimal observer gain. A DCDC converter case study in simulation is used to illustrate the utilized techniques and the simulation results prove the effectiveness.
\end{abstract}

\section{INTRODUCTION}

States estimators play an important role in the reliability assessment for critical engineering applications [1]. The latter include power electronic circuits that are as complex as their control process being subject to permanent and intermittent faults [2]. Therefore, predicting such faults has become critical for providing high operational availability of electronics-rich systems. However, the intermittent faults are unpredictable in such systems [3]. Furthermore, this paper is focused on faults that are particularly described by slow degradation profiles. The latter affect the performance of the system due to its harsh operating conditions, such as electrical and thermal overstress [4]. Consequently, advanced preventive maintenance and monitoring shall be required for the Prognostics and Health Management (PHM). Since the prognostics techniques could be considered as one of the most challenging aspects [5], not all their aspects have been covered yet [6]. Whereas, the basic system monitoring consists of historical data collection and storage, the prognosis aims to provide an on-line prediction of the RUL

This work is co-funded by European Union and Normandy Region. Europe is involved in Normandy through the European Funds for Regional Development.

†Advanced Control Systems Group, Automatic Control Department, Universitat Politècnica de Catalunya (UPC), Campus de Terrassa, Rambla Sant Nebridi, 10, 08222, Spain

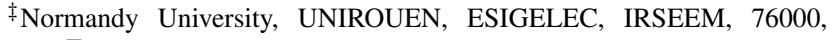
Rouen, France

†, Ahmad Al-Mohamad: ahmad.al.mohamad@upc.edu, ahmad.al-mohamadeuniv-rouen. fr

†Vicenç Puig: vicenc.puig@upc.edu

$\ddagger$ Ghaleb Hoblos: Ghaleb. hoblos@esigelec.fr of the power electronic devices [7]. Hence, historical data could be aggregated from Accelerated aGing Experiments (AGE), also known as run-until-failure tests, to characterize all the possible degradation aspects.

Previous works in [2] and [8] have shown the estimation ability of Extended Kalman Filter (EKF)-based estimators in the presence of Gaussian uncertainties. Two Physicsof-Failure (PoF)-based techniques have been associated to track the evolution of the most essential degraded parameters, MOSFET and Electrolytic Capacitors (ECaps). Hence, the state-space model of the system will consist of discrete-time with varying parameters characteristics. Furthermore, the huge industrial applications and critical power electronic devices in autonomous vehicles and airplanes imply economical constraints as well as risky decision making in terms of preventive maintenance. The burden on RUL forecasting requires reliable estimation base for an accurate prediction.

Explicitly, from a mathematical point of view, the stochastic estimators as KFs deal with linear systems in presence of Gaussian white noises. Hence, for nonlinear systems, EKF can be applied by computing the covariances using the Jacobian of nonlinear functions. Additionally, the prediction matrices are normally updated by real-time measurements with stochastic noises [9], [10]. Alternatively, most of real applications are subject to non-Gaussian noises. Thus, the set-membership does not require any assumption about the probability distributions and rely on unknown-but-bounded uncertainties. Similarly to the interval approach [11], both of them belong to the family of deterministic approaches [12]. Furthermore, there exists a few geometric sets for its applications such as ellipsoidal state sets, polyhedrons and zonotopes [13], [9]. Exploiting the representation of the zonotopic-sets clearly shows its computing simplicity when propagating the uncertainty sets.

It is interesting to consider the optimal gain design with the hybridization between KF and zonotopes proposed [13] to deal with discrete nonlinear parameter varying systems using the so-called ZEKF. Hence, following the logic of EKF and linearizing using the Jacobian method by applying the optimal criterion of the zonotopic gain, bounding sets for states and parameters in zonotopic shapes can be obtained for the degraded DC-DC converter application.

This paper adopts the ZEKF technique for parameter estimation of dynamical systems with slow variations of parameters. Thus, the novelty is based on taking advantage of the estimated zonotopic sets for the calculation of RUL 
prediction without a prior knowledge of the degradation model.

This paper is organized as follows. Section II represents the problem statement and its formulation. The ZEKF algorithm for estimation is explained in section III. In Section IV, the proposed ZEKF-based RUL forecasting approach is described. Section V is dedicated to present the DC-DC converter case study and simulation results. Finally, conclusions and perspectives are drawn in Section VI.

\section{PROBLEM STATEMENT}

Prognostics techniques intend to forecast the RUL either by implementing model-based methodologies or data-driven, depending on the complexity and the probability of faults. Although continuous monitoring and online surveying could maintain the system operations, it would lead to cut-offs due to optimistic protection or expensive maintenance due to over-protection. Essentially, the PoF of the power electronic devices have taken an interesting phase for model-based prognostics by providing empirical degradation model which can be analytically applied. Although the aforementioned studies deal with component-level prognostics, the correlation of the power electronic components in the system points out to a higher level prognosis. Hence, for systemlevel prognosis, the RUL of the system could be based on the EoL of the most affected components in the system. Hence, ZEKF approach has been adopted for the estimation of states and parameters in addition to the RUL prediction.

Figure 1 illustrates the general vision of the problem statement. As shown, three main stages characterize the system: degradation modeling, ZEKF-based states and parameters estimation and RUL prediction bounds.

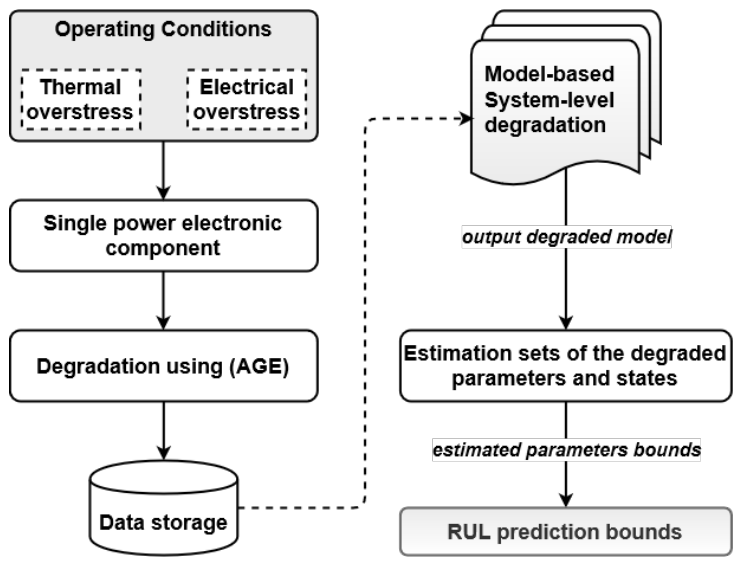

Fig. 1. Problem statement of the PHM architecture.

\section{Model Structure}

Consider a nonlinear discrete-time state-space model where its dynamics are function of a time-varying parameter $\delta$ :

$$
\begin{cases}x_{k+1} & =A_{k} x_{k}+B_{k} u_{k}+E_{\omega} \omega_{k}, \\ y_{k} & =C_{k} x_{k}+D_{k} u_{k}+E_{v} v_{k}\end{cases}
$$

where, $A_{k}, B_{k}, C_{k}$ and $D_{k}$ are functions of the time-varying parameter $\delta_{k}$ which affects the dynamics of the model due to its degradation as follows: $A\left(\delta_{k}\right), B\left(\delta_{k}\right), C\left(\delta_{k}\right)$ and $D\left(\delta_{k}\right)$. $x_{k} \in \mathbb{R}^{n_{x}}$ represents the state vectors augmented with the parameter $\delta_{k} . y_{k} \in \mathbb{R}^{n_{y}}$ is the output vector and $u_{k} \in \mathbb{R}^{n_{u}}$ denotes the inputs. $A_{k} \in \mathbb{R}^{n_{x} \times n_{x}}, B_{k} \in \mathbb{R}^{n_{x} \times n_{u}}, C_{k} \in$ $\mathbb{R}^{n_{y} \times n_{x}}$ and $D_{k} \in \mathbb{R}^{n_{y} \times n_{u}}$ are the state, input, output and feed-through matrices respectively, with $\mathbb{R}^{n}$ is a set of $\mathrm{n}$ dimensional real numbers. Additionally, $\omega_{k} \in \mathbb{R}^{n_{x}}$ and $v_{k}$ $\in \mathbb{R}^{n_{y}}$ are the process and measurement noises respectively which are assumed to be unknown but bounded. Both $E_{\omega}$ and $E_{v}$ represent the distribution matrices for the process and measurement noises.

Moreover, $x_{0} \in Z\left\langle c_{0}, H_{0}\right\rangle \subset \mathbb{R}^{n_{x}}$. Where, $Z\langle c, H\rangle \subset \mathbb{R}^{n}$, is a zonotope with center $c \in \mathbb{R}^{n}$ and a generator matrix $H \in \mathbb{R}^{n \times q} . \omega=\left\langle c_{\omega}, H_{\omega}\right\rangle$ and $v=\left\langle c_{v}, H_{v}\right\rangle$ are assumed to be bounded by the unitary hypercube zonotope centered at 0, as follows: [13], [12]

$$
\forall k \geq 0, \quad \omega_{k} \in[-1,1]^{n_{\omega}} \quad \text { and } \quad v_{k} \in[-1,1]^{n_{v}},
$$

where, $k \in \mathbb{N}$ denotes the discrete time-step. Henceforth, for the sake of simplification, the state-space matrices with timevarying parameters $A\left(\delta_{k}\right), B\left(\delta_{k}\right), C\left(\delta_{k}\right)$ and $D\left(\delta_{k}\right)$ will be represented, in the following, by $A_{k}, B_{k}, C_{k}$ and $D_{k}$ respectively.

\section{ZEKF FOR STATES AND PARAMETERS SETS ESTIMATION}

\section{A. Zonotopic Observer Strucutre}

The design of a zonotopic observer consists of the center and the generator matrices of the zonotopic sets. Unlike the KF-based observers, unknown-but-bounded noises will be considered instead of the Gaussian probability density functions. Hence, the Jacobian will be calculated for the nonlinear functions to rhyme with the EKF original algorithm. Finally, the bounding sets of the ZEKF are as follows:

$$
\begin{aligned}
c_{k+1} & =\left(A_{k}-G_{k} C_{k}\right) c_{k}+\left(B_{k}-G_{k} D_{k}\right) u_{k}+G_{k} y_{k}, \\
H_{k+1} & =\left[\begin{array}{lll}
\left(A_{k}-G_{k} C_{k}\right) \bar{H} & E_{\omega} & -G_{k} F_{v}
\end{array}\right],
\end{aligned}
$$

where, $\bar{H}=\downarrow_{\mathrm{q}} H_{k}$ and $\downarrow_{\mathrm{q}}$ represents the reduction matrix. $G_{k}$ represents the time-variant observer gain.

The step-ahead state $x_{k+1}$ satisfies $x \in Z\langle c, H\rangle, \forall k \geq 0$ :

$$
x_{k+1}=A_{k} x_{k}+B_{k} u_{k}+E_{\omega} \omega_{k}+G_{k}\left(Y_{k}-\hat{y}_{k}\right) \text {, }
$$

where, $Y$ is the measured output vector and $\hat{y}$ is its estimation and the linearization process is done as follows:

$$
A_{k}=\left.\frac{\partial f}{\partial x}\right|_{x_{k}, u_{k}}, B_{k}=\left.\frac{\partial f}{\partial u}\right|_{x_{k}, u_{k}} \text { and } C_{k}=\left.\frac{\partial g}{\partial x}\right|_{x_{k}, u_{k}} \text {. }
$$

\section{B. Optimal Gain}

Computing the optimal gain $G^{*}$ can be achieved by minimizing the $F_{W}$-radius, which is a criterion introduced with the previously mentioned reduction operator. It also has the same size of the zonotope $Z\left\langle c_{k+1}, H_{k+1}\right\rangle$ as referred in [13]. As defined in this reference, the $F_{W}$-radius of a zonotope $Z$ is the Frobenius norm of $H:\|\langle c, H\rangle\|_{F}=\|H\|_{F}$. 
Moreover, the obtained optimal gain $G^{*}$ can lead to equations similar to those of the KF.

$$
\begin{aligned}
G^{*} & =A K^{*}, \\
K^{*} & =L S^{-1}, \quad L=\bar{P} C^{T}, \quad S=C \bar{P} C^{T}+Q_{\omega},
\end{aligned}
$$

where the covariation matrices are as follows,

$$
\begin{aligned}
& P=H H^{T}, \quad Q_{\omega}=F F^{T}, \\
& \bar{P}=\bar{H} \bar{H}^{T}=\operatorname{cov}\left(\downarrow_{q} H\right) \text { is the covariation of } Z .
\end{aligned}
$$

The author also states that minimizing the $F$-radius of a zonotope $Z\left\langle c_{k+1}, H_{k+1}\right\rangle$ is equivalent to minimizing the trace of its covariation $P_{k+1}=H_{k+1} H_{k+1}^{T}$.

\section{ZEKF Estimation Algorithm}

Considering the nonlinear discrete-time parameter varying system in (1). The estimation algorithm keeps the structure of the EKF filtering equations. However, the new optimal gain $G^{*}$ is introduced to the time-varying state equation in (4). Consequently, by implementing the zonotopic equations in (3), the resulted states are bounded in a zonotope. Here follows the ZEKF algorithm, after affecting the system parameters by the empirical degradation models:

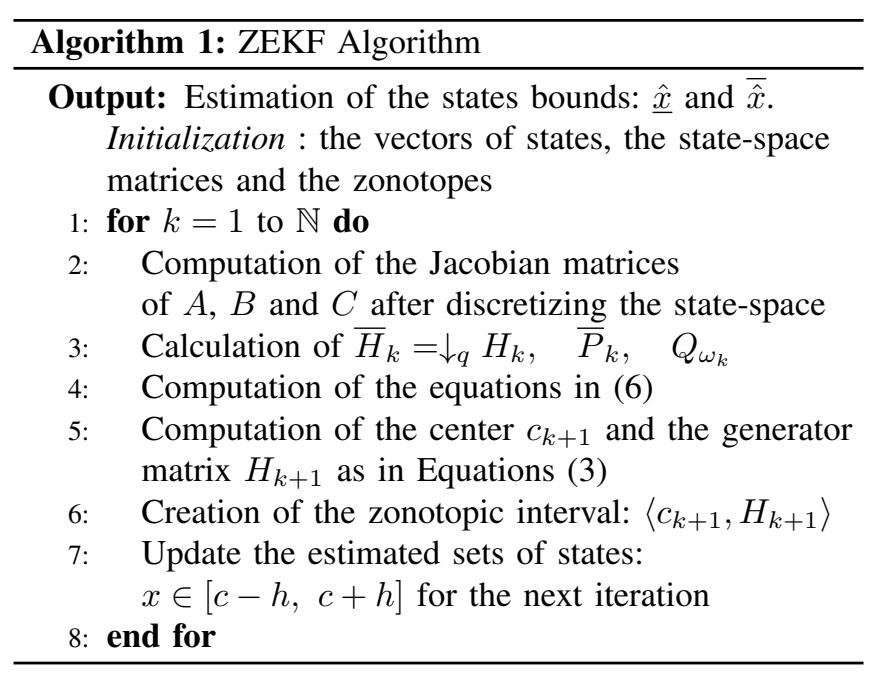

The interval for estimated states, $[\underline{x}, \bar{x}]$ can be obtained from $H$, by calculating the interval hull of the zonotope allowing to determine $h$.

\section{Prognostics ApProACH}

\section{A. Proposed PHM Architecture}

In broad, the reliability assessment requires trustworthy and efficient estimation. Given that the characteristics of the power electronic devices are different, the treatment of every component in the system would be a complex burden. Consequently, the proposed approach depends on the historical data as references of the probabilities of failure of the components considering that have different degradation manifolds due to their PoF. Thus, a system-level approach should consider the estimation of all the degraded components in the system. Whereas, the whole system could fail if a power electronic switch or a capacitor degrade to a certain limit. Hence, the extensive AGE have analyzed the behavior of the aforementioned components in order to define their EoL or the threshold which imposes urgent maintenance or indicates a complete failure.

Moreover, the bulk of components are operating in the same environmental conditions. Thus, with the help of the AGE data, the simulation of the empirical models have defined degradation rate with the inter-effects of the components in such systems.

Consequently, the proposed prediction method relates the RUL decision of the system to the component which is degrading the most. Thus, the parameter estimation is the decision base of the RUL of the whole system in an accepted interval. Critical applications and pessimistic usages could make decisions concerning the maintenance in accordance with the online measurement of the lower bound of the RUL. While, in contrary for optimistic usages that can go up to the higher bound of the RUL.

Figure 2 illustrates the whole proposed PHM algorithm which is divided into three main stages to deal with the different algorithmic procedures:

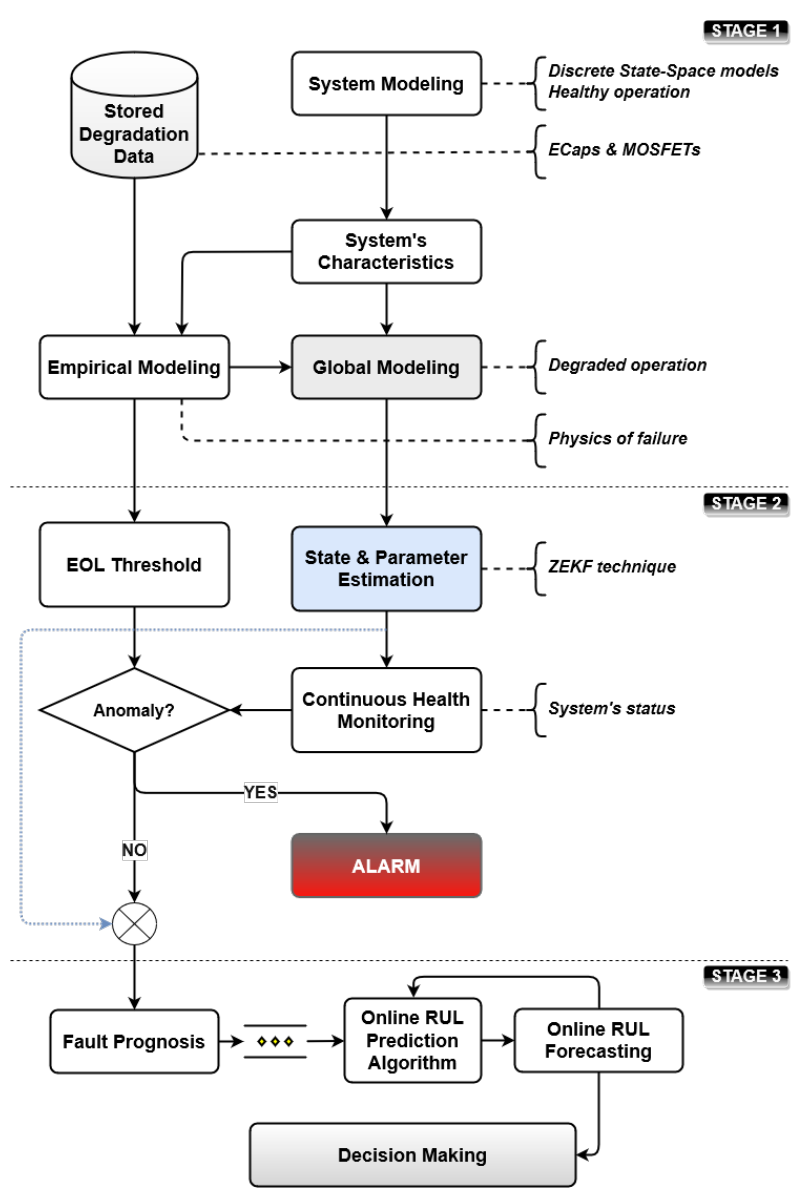

Fig. 2. Proposed PHM flowchart. 


\section{Stage 1: System modeling and features extraction}

- The modeling of the plant comes in the healthy operating mode as a first step of the analytical study. Thus, it is crucial to specify the sensors and the measurement devices precision and noise power for further applications.

- The system characteristics are important to extract the environmental working conditions, the limitations of the parameters in order to establish a study about their physics of failure.

- The AGE help building a database used for statistical analysis of the behaviour of the power electronic components. Thus, the degradation analysis is numerically calculated for the next step. The degradation data are available online from the data repository of the prognostics center of NASA.

- The previous step leads to generalize degradation equations for each and every tested electronic components. Hence, empirical models infect the healthy operation mode to create the degraded state-space models of the whole system.

- Finally in stage 1 of prognostics study, a global model has to be generated either using model-based or datadriven techniques for the simulation study of the PHM.

Stage 2: Parameters estimation and health monitoring

- The ZEKF technique is responsible of estimating the sets of states of the global model for the $k+1$ iteration. In parallel, the slow-variation are also estimated using the advanced filtering techniques due to the difficulty of them to be directly measured.

- The system is continuously monitored to detect any sudden fault, allocate it and then isolate it. This process is traditionally known as fault diagnosis and it is based on the estimation done in the previous step. in case of fault, the system will take decision by a human interface or it could be self-cleared depending on its intensity. However, for the non-faulty condition, the prognosis algorithm will take place in order to estimate the RUL of the system.

\section{Stage 3: RUL prognosis}

Independently of the degradation knowledge, the RUL forecasting algorithm considers the zonotopic parameter sets as inputs. At each time step, the fault prognosis algorithm fits the measured parameter zonotope with a polynomial equation which updates its variables at each iteration. A linear EoL-RUL equation is optimistically assumed in the prognosis profile. Moreover, the algorithm runs in online mode and it converges at each iteration. It is also assumed that the component break-through threshold is the stopping criterion, the EoL of the system. Therefore, the expected results are intervals of RUL function of operating time, where the trusted region lies inside. The simulation results in Section $\mathrm{V}$, numerically explains the robustness of this proposed technique. Algorithm 2 presents the procedure used to compute the intervals of the RUL.

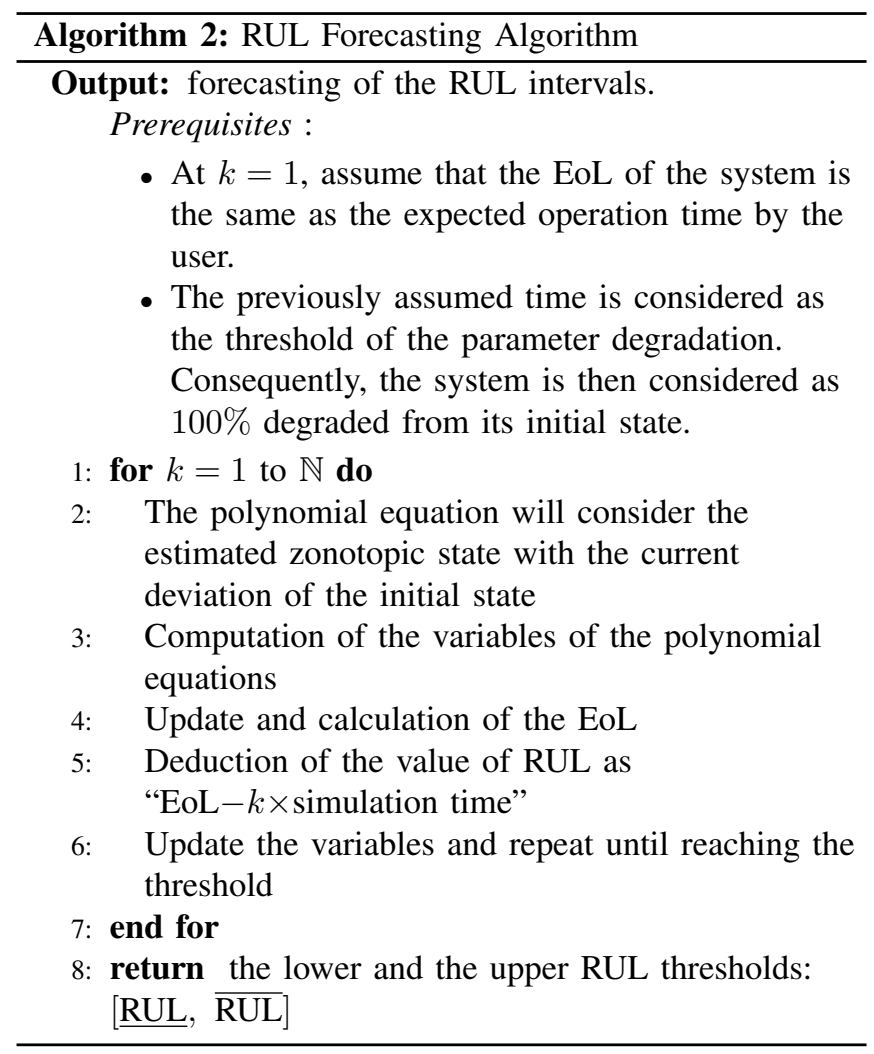

Moreover, to analyze the performance of the RUL forecasting technique, It is proposed to evaluate the Prognosis Horizon $(\mathrm{PH})$ for the reliability and the Relative Accuracy (RA) [14]:

$$
\mathrm{PH}_{k}=\mathrm{EoL}-k,
$$

where, PH is one of a few Prognostic Performance Metrics (PPM) and it is calculated to analyze if the RUL algorithm meets the desired accuracy around the EoL. Thus, the RA is another PPM used to quantify the accuracy of the algorithm at any measurement time.

$$
\mathrm{RA}_{\%}=100-\frac{\left|\mathrm{RUL}_{k}^{\text {pred }}-\mathrm{RUL}_{k}\right|}{\mathrm{RUL}_{k}} \times 100,
$$

the higher the RA percentage is, the more the prediction with respect to RUL is accurate.

\section{DC-DC Converter Case Study}

The switch-mode power converters are widely used in electric vehicles as bi-directional buck-boost converters. Thus, they experience environmental as well as operational stress such as heat, current spikes and voltage surges [4]. Hence, these factors affect the proper operating conditions of the converters and deteriorate the efficiency of the system including the internal components. Therefore, the reliability of the system would not be trustworthy in huge industrial applications such as nuclear power plants and autonomous vehicles. Consequently, the PHM would prevent these systems from unnecessary and expensive maintenance [15]. 


\section{A. Boost Converter Modeling}

A $30 \mathrm{~kW}$ DC-DC converter would reflect a perfect application of prognosis application and is designed as shown in Figure 3. Due to space limitation constraints, only the boost application is explained in this paper. Refer to [16] for more detailed modeling of the boost converter.

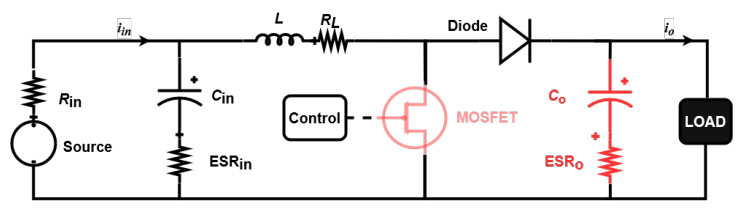

Fig. 3. Boost converter circuit diagram.

Furthermore, the switching power devices and ECaps are the components more prone to present faults [2]. Thus, in [8], the degradation estimation of MOSFETs for the buckboost converter, has been explicitly explained as well as the ECaps in [2] using EKF-based techniques. However, the empirical degradation of the output ECap in the boost operation has been implemented to engage the ZEKF for the estimation and the prediction processes. Note that the Jacobian calculations should be done for each of the submodels first. Then, the average discrete matrices can be calculated. Moreover, the augmented states vector becomes $x_{k}^{\text {aug }}=$ $\left[v_{C_{\text {in }}} ; \quad i_{L} ; \quad v_{C_{\mathrm{o}}} ; \delta_{k}\right]$ and the augmented equations as in (1) are then implemented with $15 \mathrm{khZ}$ sampling frequency. Hence, the dimensions of the augmented state-space matrices change as follows:

$\operatorname{dim}\left[A_{\mid \text {aug }}(\cdot)\right]=4 \times 4, \operatorname{dim}\left[B_{\mid \operatorname{aug}}(\cdot)\right]=4 \times 2$, $\operatorname{dim}\left[C_{\mid \text {aug }}(\cdot)\right]=2 \times 4$ and $\operatorname{dim}\left[D_{\mid \text {aug }}(\cdot)\right]=2 \times 2$, and the augmented matrices become:

$$
\begin{aligned}
A_{\text {|aug }} & =\left[\begin{array}{cc}
A & \{0\} \\
\{0\} & 1
\end{array}\right], & B_{\text {|aug }} & =\left[\begin{array}{c}
B \\
\{0\}
\end{array}\right] \\
C_{\text {|aug }} & =\left[\begin{array}{ll}
C & \{0\}
\end{array}\right], & D_{\text {|aug }} & =[D] .
\end{aligned}
$$

\section{B. Degradation Modeling}

The PoF of the power electronic devices are explicitly explained in [2], [8], [16]. Thus, for simulation purposes, the empirical models of the output capacitor and its ESR were numerically fitted as exponential functions.

The following equation represents the degraded capacitance value which is used to degrade the models to simulate real life measurements from experimental open-source data sets provided by NASA PCoE:

$$
C_{\text {deg }}(t)=C_{0}\left(1-C_{\%}(t)\right) / 100,
$$

where, $t$ is the continuous time and the capacitance percentage loss is expressed in the following equation:

$$
C_{\%}(t)=e^{\alpha t}+\beta,
$$

with, $\alpha=0.0163$ and $\beta=-0.8398$.

Unlike the capacitance, the ESR shows an increase of its value due to degradation. Since, the AGE have shown that the capacitor is faulty for $20 \%$ of capacitance degradation [4], the ESR is then allowed to increase by $58 \%$ from its initial value. Thus, the thresholds are defined by the experimental values.

Below is the equation of the ESR increase:

$$
\mathrm{ESR}_{\uparrow}=a_{1} e^{b_{1} t}+c_{1} e^{d_{1} t},
$$

with, $a_{1}=21.91, b_{1}=0.005117, c_{1}=-22.2$ and $d_{1}=$ -0.02211 .

It should be noted that these equations are only used to simulate the degradation of the system. Whereas, the RUL prediction algorithm computes the variables (e.g. $a_{1}, b_{1}, \ldots$ ) and predicts its shape to create the RUL zonotopic intervals.

\section{Simulation Results}

Algorithm 1 of ZEKF estimation is mainly implemented for states and parameters estimation in zonotopic sets. The states vector is initialized with random values such as 50, 100 and 200 for input capacitance voltage, inductor current and output capacitor voltage respectively while their ideal values without degradation are $200 \mathrm{~V}, 150 \mathrm{~A}$ and $300 \mathrm{~V}$ in the same order. Thus, the input voltage is set to 200 $\mathrm{V}$ and the output current is $100 \mathrm{~A}$. Hence, the state-space matrices at $k=1$ are computed with the initial value of the degraded parameter $\delta_{0}=\mathrm{ESR}_{0}=80 \mathrm{~m} \Omega$, which is in this simulation the ESR of the output capacitor of the converter. It should be noted, that the degradation models of the components are extracted from AGE and then fitted in empirical models. However, in this paper they are only used for comparison and not for estimation nor prediction. The only two affected states are the output voltage and the augmented parameter $\delta$. The output voltage shows a slow degradation profile as shown in previous work [2], while the ESR shows an increasing trend as shown in the Figure 4 with the estimated bounds of the ESR evolution. First of all, the empirical degradation model is illustrated in blue as a reference to compare with the estimation. Thus, the red lines correspond to the lower and upper bounds of the ZEKF-based parameter estimation. As illustrated, the estimated parameter ESR in green $\in\left[\mathrm{ESR}_{0}, \overline{\mathrm{ESR}}_{\mathrm{o}}\right]$. Thus, the estimated ESR remains in the zonotopic intervals during the accelerated simulation cycles.

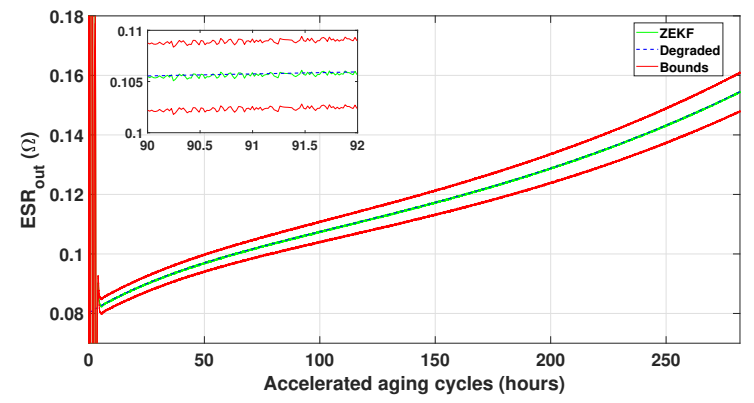

Fig. 4. $\mathrm{ESR}_{o}$ estimation during capacitor degradation with the lower and upper bounds.

Moreover, the essential results of algorithm 2 of this approach are demonstrated in Figure 5. At each time-step, the 
polynomial fit of the earlier estimation computes the current RUL interval of the system, based on the assumptions noted in the same algorithm. At any measurement time, the RUL lies between the predicted bounds and the decision would be made depending on the application.

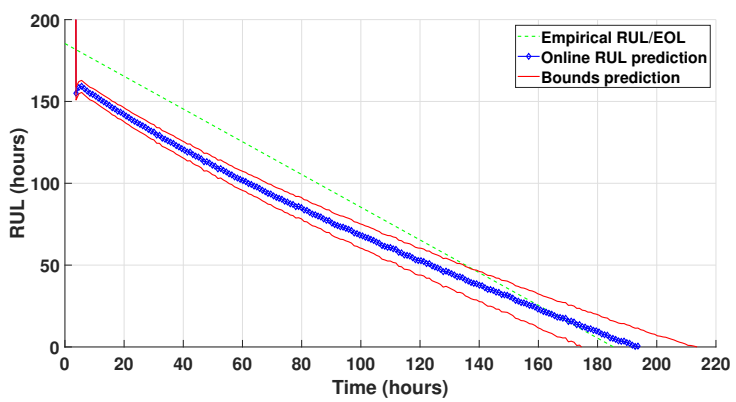

Fig. 5. RUL forecasting intervals of the system during capacitor degradation.

It is worthwhile noting that the state estimate results are not shown in this paper because the focus is on the RUL approach which is based on the parameter estimate and not the states.

Furthermore, testing the accuracy of the RUL prediction is calculated using Eq. (9) and the results are shown in Figure 6. Hence, a high percentage of RA lies between $80 \%$ and $100 \%$ in the steady prediction zone. Consequently, this means that the prediction process is optimistic with respect to the empirical model.

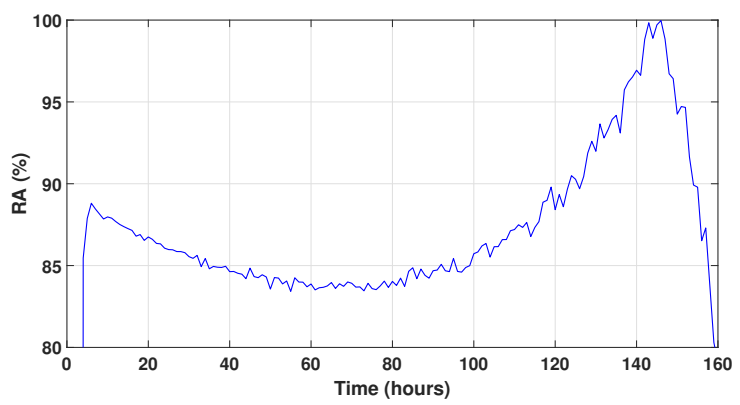

Fig. 6. The relative accuracy of the RUL prediction with respect to the empirical model.

\section{CONCLUSIONS}

A new RUL forecasting methodology has been proposed in this paper to point out the integration of the ZEKF estimation with prognostics vision. The estimation process of nonlinear time-variant system has been achieved by augmenting the states with a varying parameter and applying the EKF with an optimal gain in order to compute the zonotopic sets of estimates. Besides, the failure mechanisms of the power electronic devices play an essential role in model-based computing along with the AGE techniques. Straightforward into the problem, the proposed algorithm has taken advantage of the zonotopes for the prognostics approach. The main contribution in this paper is clearly shown in the results where the RUL bounds are predicted with no prior knowledge of which parameter is degrading. Consequently, this methodology could enhance the reliability of predictions for electronics-rich systems with unknown-but-bounded noises. Additionally, limiting the degradation effects could be a possible solution to minimize the damage and then the costly maintenance. Finally, the simulation shows high accuracy prediction enhanced by the ZEKF to deal with such systems.

\section{REFERENCES}

[1] Jitao Li, Zhenhua Wang, Wenhan Zhang, Tarek Raïssi, Yi Shen, Interval observer design for continuous-time linear parameter-varying systems, Systems \& Control Letters, Volume 134, 2019, 104541, ISSN 0167-6911, https://doi.org/10.1016/j.sysconle.2019.104541.

[2] A. Al-Mohamad, G. Hoblos and V. Puig, "A Model-Based Prognostics Approach for RUL Forecasting of a Degraded DC-DC Converter," 2019 4th Conference on Control and Fault Tolerant Systems (SysTol), Casablanca, Morocco, 2019, pp. 312-318.

[3] Pecht M. (2012) A Prognostics and Health Management for Information and Electronics-Rich Systems. In: Mathew J., Ma L., Tan A., Weijnen M., Lee J. (eds) Engineering Asset Management and Infrastructure Sustainability. Springer, London

[4] C. S. Kulkarni, J. R. Celaya, G. Biswas and K. Goebel, "Prognostics of Power Electronics, methods and validation experiments," 2012 IEEE AUTOTESTCON Proceedings, Anaheim, CA, 2012, pp. 194-199.

[5] Elattar, H.M., Elminir, H.K. \& Riad, A.M. Prognostics: a literature review. Complex Intell. Syst. 2, 125-154 (2016). https://doi.org/10.1007/s40747-016-0019-3

[6] Yousfi B, Raïssi T, Amairi M, Aoun M. Set-membership methodology for model-based prognosis. ISA Transactions. 2017 Jan;66:216-225. DOI: 10.1016/j.isatra.2016.09.025.

[7] E. I. Robinson, J. Marzat \& T. Raïssi (2019) Prognosis of uncertain linear time-invariant discrete systems using unknown input interval observer, International Journal of Control, DOI: 10.1080/00207179.2019.1648854

[8] A. Alyakhni, A. Al-Mohamad and G. Hoblos, "Joint Estimation of MOSFET Degradation in a DC-DC Converter Using Extended Kalman Filter," 2019 4th Conference on Control and Fault Tolerant Systems (SysTol), Casablanca, Morocco, 2019, pp. 319-324.

[9] Wang, Y., Puig, V. Zonotopic extended Kalman filter and fault detection of discrete-time nonlinear systems applied to a quadrotor helicopter. A: International Conference on Control and Fault-Tolerant Systems. "SYSTOL 2016 - 3rd Conference on Control and FaultTolerant Systems, Barcelona, Spain, Sept. 7-9, 2016, proceedings book". Barcelona: IEEE Press, 2016, p. 367-373.

[10] L. Sun, H. Alkhatib, B. Kargoll, V. Kreinovich, and I. Neumann, "Ellipsoidal and Gaussian Kalman Filter Model for Discrete-Time Nonlinear Systems," Mathematics, vol. 7, no. 12, p. 1168, Dec. 2019.

[11] J.L. Gouzé, A. Rapaport, M.Z. Hadj-Sadok, Interval observers for uncertain biological systems, Ecological Modelling, Volume 133, Issues 1-2, 2000, Pages 45-56, ISSN 0304-3800, https://doi.org/10.1016/S0304-3800(00)00279-9.

[12] Masoud Pourasghar, Christophe Combastel, Vicenç Puig, Carlos Ocampo-Martinez, FD-ZKF: A Zonotopic Kalman Filter optimizing fault detection rather than state estimation, Journal of Process Control, Volume 73, 2019, Pages 89-102, ISSN 0959-1524, https://doi.org/10.1016/j.jprocont.2018.12.003.

[13] Christophe Combastel, Zonotopes and Kalman observers: Gain optimality under distinct uncertainty paradigms and robust convergence, Automatica, Volume 55, 2015, Pages 265-273, ISSN 0005-1098, https://doi.org/10.1016/j.automatica.2015.03.008.

[14] S. Benmoussa and M. A. Djeziri, "Remaining useful life estimation without needing for prior knowledge of the degradation features," in IET Science, Measurement \& Technology, vol. 11, no. 8, pp. 10711078, 112017.

[15] J. K. Mann, S. Perinpanayagam and I. Jennions, "Aging Detection Capability for Switch-Mode Power Converters," in IEEE Transactions on Industrial Electronics, vol. 63, no. 5, pp. 3216-3227, May 2016

[16] Ahmad Al-Mohamad, Ghaleb Hoblos, Vicenç Puig, "A hybrid system-level prognostics approach with online RUL forecasting for electronics-rich systems with unknown degradation behaviors", Microelectronics Reliability, 2020, https://doi.org/10.1016/j.microrel.2020.113676 